\title{
BMJ Open Study protocol for the COPE study: COVID-19 in Older PEople: the influence of frailty and multimorbidity on survival. A multicentre, European observational study
}

\author{
Angeline Price (10 , ${ }^{1}$ Fenella Barlow-Pay, ${ }^{2}$ Siobhan Duffy, ${ }^{3}$ Lyndsay Pearce, ${ }^{4}$ \\ Arturo Vilches-Moraga, ${ }^{1}$ Susan Moug, ${ }^{3,5}$ Terry Quinn, ${ }^{6}$ Michael Stechman, ${ }^{7}$ \\ Philip Braude, ${ }^{8}$ Emma Mitchell, ${ }^{8}$ Phyo Kyaw Myint (D) , ${ }^{9}$ Alessia Verduri, ${ }^{10}$ \\ Kathryn McCarthy, ${ }^{11}$ Ben Carter, ${ }^{12,13}$ Jonathan Hewitt (D) , ${ }^{14}$ COPE Study \\ Collaborators
}

To cite: Price A, Barlow-Pay F, Duffy S, et al. Study protocol for the COPE study: COVID-19 in Older PEople: the influence of frailty and multimorbidity on survival. A multicentre, European observational study. BMJ Open 2020;10:e040569. doi:10.1136/ bmjopen-2020-040569

- Prepublication history and additional material for this paper are available online. To view please visit the journal (http:// dx.doi.org/10.1136/bmjopen2020-040569).

Received 18 May 2020 Revised 28 July 2020 Accepted 11 September 2020

Check for updates

(C) Author(s) (or their employer(s)) 2020. Re-use permitted under CC BY-NC. No commercial re-use. See rights and permissions. Published by BMJ.

For numbered affiliations see end of article.

Correspondence to

Angeline Price;

angeline.price@srft.nhs.uk

\section{ABSTRACT}

Introduction This protocol describes an observational study which set out to assess whether frailty and/or multimorbidity correlates with short-term and mediumterm outcomes in patients diagnosed with COVID-19 in a European, multicentre setting.

Methods and analysis Over a 3-month period we aim to recruit a minimum of 500 patients across 10 hospital sites, collecting baseline data including: patient demographics; presence of comorbidities; relevant blood tests on admission; prescription of ACE inhibitors/angiotensin receptor blockers/non-steroidal anti-inflammatory drugs/ immunosuppressants; smoking status; Clinical Frailty Score (CFS); length of hospital stay; mortality and readmission. All patients receiving inpatient hospital care $>18$ years who receive a diagnosis of COVID-19 are eligible for inclusion. Long-term follow-up at 6 and 12 months is planned. This will assess frailty, quality of life and medical complications. Our primary analysis will be short-term and long-term mortality by CFS, adjusted for age (18-64, 65-80 and $>80$ ) and gender. We will carry out a secondary analysis of the primary outcome by including additional clinical mediators which are determined statistically important using a likelihood ratio test. All analyses will be presented as crude and adjusted HR and OR with associated 95\% Cls and $p$ values.

Ethics and dissemination This study has been registered, reviewed and approved by the following: Health Research Authority (20/HRA1898); Ethics Committee of Hospital Policlinico Modena, Italy (369/2020/0SS/AOUM0); Health and Care Research Permissions Service, Wales; and NHS Research Scotland Permissions Co-ordinating Centre, Scotland. All participating units obtained approval from their local Research and Development department consistent with the guidance from their relevant national organisation.

Data will be reported as a whole cohort. This project will be submitted for presentation at a national or international surgical and geriatric conference. Manuscript(s) will be prepared following the close of the project.
Strengths and limitations of this study

This is a large, multicentre study collecting data from throughout Europe.

- Data are collected by a network of clinicians with an established track record of research in frailty.

- Data collection is not entirely prospective. Some will be collected retrospectively.

- Targeted data collection was carried out.

\section{INTRODUCTION}

SARS-CoV-2 or COVID-19 was identified in 2019 as a novel strain of pneumonia inducing virus. Coronaviruses have been identified since the 1960 s, with other strains including SARS-related coronavirus in 2003 and Middle East respiratory syndrome-related coronavirus in 2012 causing significant human harm and global spread. COVID-19 has now been declared as a current worldwide pandemic ${ }^{1}$ with predominant symptoms including: fever, cough and shortness of breath. The majority of people with COVID-19 have mild symptoms $(81 \%)$, some develop severe illness requiring oxygen therapy $(14 \%)$, with a smaller number requiring mechanical ventilation and/or intubation (5\%). Early on in the pandemic, the most commonly reported presentation in severe COVID-19 was bilateral pneumonia. ${ }^{2}$ Emerging literature has identified delirium, gastrointestinal disturbance and falls as predominant clinical signs in older, frail patients ${ }^{34}$

Certain risk factors have been identified as predisposing patients to poor outcomes such as hospital admission, need for ventilation and 
subsequent death. Individuals with risk factors including older age and comorbidities such as hypertension, cardiovascular disease and diabetes mellitus have an increased risk of severe disease poor outcomes in both COVID-19 and non-COVID-19 populations. ${ }^{56}$ There has been speculation that drugs such as ACE inhibitors (ACEi) (eg, ramipril, lisinopril, perindopril) and angiotensin receptor blockers (ARB) (losartan, candesartan), commonly taken by patients with hypertension, heart failure and diabetes mellitus, might increase susceptibility to coronavirus infection, ${ }^{7}$ as might other groups of drugs including non-steroidal anti-inflammatory drugs (NSAIDs) and immunosuppressants. These could be simple association rather than causation given the common use of pharmacotherapy such as ACEi and ARBs in multimorbid individuals. Chronic musculoskeletal and respiratory diseases are common in our ageing population and as a result there is a high usage of NSAIDs (either prescribed or over the counter) and long-term steroids. There is a theoretical possibility that immunosuppressants, such as steroids or other agents, used to treat other conditions (eg, renal disease, vasculitis, inflammatory bowel disease, transplant), confer benefit by modifying the host immune response to the virus resulting in less severe illness. ${ }^{8}$

Current professional guidelines recommend continuing these drugs. ${ }^{910}$ This is based on a lack of available evidence that would clearly support withholding these medications during the COVID-19 pandemic. By collecting data on these medications and their relationship to COVID-19 outcomes, we aim to improve on the current deficiency in evidence on whether it is safe to continue these medications, or whether they should be withdrawn.

Frailty is recognised as a potential contributing factor to poor outcomes in COVID-19. Frailty is characterised by reduced physiological reserve ${ }^{11}$ and defined as 'a medical syndrome with multiple causes and contributors that is characterised by diminished strength, endurance and reduced physiological function that increases an individual's vulnerability for developing increased dependency or death'. ${ }^{12}$ The frailty phenotype model categorises individuals into frail, prefrail or not frail according to the presence of weight loss, self-reported exhaustion, low energy expenditure, slow gait speed and weak grip strength. ${ }^{13}$ The cumulative deficit model is validated to grade frailty in a person by identifying disability and comorbidity. ${ }^{14}$ Frailty is associated with greater age, but not exclusively, ${ }^{15}$ and has been shown to correlate with adverse clinical outcomes including length of stay, mortality and institutionalisation. This association with frailty is present in geriatric medicine and across a range of ages, specialties and interventions. ${ }^{16-19}$ As a result, hospital teams are encouraged to routinely score frailty in adults. ${ }^{20}{ }^{21}$ Frailty is more widely used than age alone as a trigger for specialist resource allocation, pathway decision aid and in shared decision-making in older patients. ${ }^{22}{ }^{23}$ Recent guidance from the National Institute for Health and Care Excellence recommended identification of frailty as part of a holistic assessment in facilitation of discussions with patients regarding critical care interventions in COVID-19. ${ }^{24}$

Despite this, and due to the novel nature of COVID-19, no information is available on how frailty affects overall outcomes. While evidence relating to factors which pose an increased risk of poor outcomes for patients with COVID-19 has begun to emerge globally, ${ }^{25-27}$ there remains a paucity of evidence relating specifically to the UK population. This study aims to provide evidence regarding some of the patient characteristics which might indicate poor outcomes, as applies to the UK population and healthcare systems. It will provide valuable information about differences in care worldwide for the benefit of patients. Emerging narrative is demonstrating tentative links between both social deprivation and ethnicity on adverse outcomes in COVID-19, ${ }^{28}$ therefore this information has been incorporated into baseline data collection in order to determine how outcomes across groups differed according to these characteristics.

Currently 10 hospitals, including one site in Italy, have joined the study. The study remains open to new recruiting sites. Following ethical approval, we will advertise recruitment through the established clinical research networks in Geriatric Medicine (GemRes, based in Birmingham and WeGen based in Wales) and via social media, notably Twitter, a platform on which a high level of interest in COVID-19-related healthcare developments has been evidenced. Other sites will be eligible to recruit subject to local approval.

\section{Aims}

To assess whether frailty and/or multimorbidity correlates with poor clinical outcomes in adult patients diagnosed with COVID-19 virus in a multicentre setting.

The following research questions will be addressed:

1. What are the outcomes for frail and/or multimorbid adults diagnosed with COVID-19, including length of hospital stay and mortality?

2. What other factors influence outcomes in this setting, including medication, source of COVID-19 (community or nosocomial), socioeconomic deprivation; ethnicity.

\section{METHODS}

\section{Study summary}

Table 1 provides the summary of the COVID-19 in Older PEople (COPE) study information.

\section{Study design and setting}

Study setting: National Health Service (NHS) hospitals in the UK and worldwide partners (who will require separate local ethical compliance) that provide emergency care for patients diagnosed with COVID-19 have been invited to participate. Our team, the Older Person Surgical Outcomes Collaborative (OPSOC) (www.opsoc. $\mathrm{eu}$ ), have widely collected data on frailty previously using routinely collected service evaluation-level data, predominantly but not exclusively focusing on surgery in the 


\section{Table 1 Study summary}

Study title

COVID-19 in Older PEople:

the influence of frailty and multimorbidity on survival

$\begin{array}{ll}\text { Short title } & \text { COPE study } \\ \text { Study design } & \text { Observational } \\ \text { Study participants } & \begin{array}{l}\text { Adults aged 18 years and older, } \\ \text { inpatients in an acute hospital } \\ \text { setting with an illness relating to } \\ \text { COVID-19 }\end{array}\end{array}$

Planned size of sample Minimum 500

$\begin{array}{ll}\text { Study sites }(n) & \text { Minimum } 10 \\ \text { Start date } & 27 \text { February 2020 }\end{array}$

Planned study period 3 months

Follow-up duration 12 months

Ethics/registration Health Research Authority (20/ number

Ethics Committee of Hospital
Policlinico Modena, Italy (369/2020/
OSS/AOUMO)
Health and Care Research
Permissions Service, Wales
NHS Research Scotland
Permissions Co-ordinating Centre,
Scotland

Research question/ To assess frailty and multimorbidity aim(s) in older adults receiving acute inpatient care with a confirmed diagnosis of COVID-19. To correlate frailty with both short-term and long-term outcomes.

A secondary research question is the effect of ACEi, ARB, nonsteroidal anti-inflammatory and immunosuppressive medications in patients with COVID-19.

A further research question is how other factors including source of COVID-19 (community vs nosocomial), socioeconomic deprivation; ethnicity and gender influence outcomes in this setting. Longer term mortality, quality of life and adverse medical outcomes.

ACEi, ACE inhibitor; ARB, angiotensin receptor blocker; COPE, COVID-19 in Older PEople.

older person. OPSOC has a proven record of conducting high-quality research and successfully publishing results relating to outcomes for older people..$^{29-32}$ OPSOC has an international reputation, related specifically to the care of the frail older person. Using well-established research networks and experience in collaborative research and frailty, we wish to explore outcomes for older patients with COVID-19 infection.

Ten hospitals are already members of the OPSOC and are actively treating patients with COVID-19-Newport,
Cardiff, Southmead Hospital Bristol, Bristol Royal Infirmary, Aberdeen Royal Infirmary, Royal Alexandra Hospital Paisley, Inverclyde Royal Infirmary, Salford Royal Hospital, Addenbrookes Hospital Cambridge, and Modena, Italy. The study will be open to other sites.

Research will be conducted using the established surgical and geriatric registrar-led research networks. ${ }^{33} 34$ The methodology for these networks is well described, but in brief the networks provide local principal investigators (PIs) who will coordinate local data collection and approvals in conjunction with Good Clinical Practice. These local leads act as a link between the local COPE team and the central Study Management Group (SMG). The SMG will provide the ethical approvals, protocol and central organisation. Ultimately the SMG will analyse data and disseminate study findings, recognising all collaborators.

\section{Study population \\ Inclusion criteria}

Any patient receiving inpatient hospital care with a clinical and/or laboratory-confirmed diagnosis of COVID-19 aged 18 years and above.

\section{Exclusion criteria}

Patients less than 18 years of age, and all patients aged 18 years and above without a clinically and/or laboratoryconfirmed diagnosis of COVID-19.

\section{Patient screening}

Patients will be screened for inclusion criteria by the local team. This will be undertaken by a range of healthcare professionals involved in direct patient care at each site. Hospital or NHS number will not be entered into this form but will be kept separately with a key sheet.

All transmission and storage of data will be encrypted and compliant with Health Insurance Portability and Accountability Act security guidelines. No patient identifiable information will be uploaded or stored on the secure database (password-protected login). Collaborators will anonymise patients by recording patient hospital numbers alongside database numbers in a separate secure spreadsheet to facilitate the collection of data locally. This database will be held within Cardiff University, Wales and stored for a minimum of 5 years.

\section{Variables collected}

Baseline data

- Age, gender, deprivation score, ethnic origin.

- Comorbidities: coronary artery disease, diabetes mellitus, hypertension and the prescription of ACEi or ARB, NSAIDs and immunosuppressive agents.

- Smoking status (never, ex-smoker and current).

- Blood tests on admission or at time of symptom development: albumin, C-reactive protein, estimated glomerular filtration rate.

- Clinical Frailty Score (CFS): 1-9 (online supplemental appendix A). 


\section{Primary outcomes}

- Short-term mortality (measured at day $7^{\& \& \&}$ and time to mortality) in frail versus non-frail patients with a COVID-19 diagnosis. \&\&

- Long-term mortality (measured at 6 and 12 months and time to mortality) in frail versus non-frail patients with a COVID-19 diagnosis. ${ }^{\& \&}$

\section{Secondary outcomes}

- Time to discharge (length of hospital stay), measured in days from time of admission and/or time of COVID-19 diagnosis to discharge.

- Effect of gender on outcomes in patients with COVID-19.

- Thirty-day readmission to local hospital. ${ }^{\&}$

- Effect of certain drug classes on outcomes in patients with COVID-19. ${ }^{\&}$

- Effect of deprivation score and ethnicity on outcomes in patients with COVID-19. ${ }^{\text {\& }}$

- Adverse outcomes and quality of life at 6 and 12 months.

${ }^{\&}$ Due to the nature and need for rapid reporting the following outcomes will be subject to later data collection and may be reported separately from the primary research findings.

${ }^{\& \&}$ Secondary analysis studies will report the all-cause time to event as the primary outcome.

Frailty assessment

We will use the Rockwood CFS (online supplemental appendix A). This has been validated for use to assess frailty in older patients. ${ }^{14}$ The score ranks from 1 to 9 with a score of $\geq 5$ being classed as frail and 9 as terminally ill, and is based on function 2 weeks prior to hospital admission. To ensure data quality each PI ensured adequate knowledge within the data collection team of frailty scoring using a short training video on how to complete the Clinical Frailty Scale 1-9.

\section{Longer term follow-up}

Follow-up is planned via telephone assessment at both 6 and 12 months. This will aim to reassess frailty using the CFS, quality of life assessments; Trauma Screening Questionnaire, ICEpop CAPability measure for Older people (ICECAP-O), Patient Health Questionnaire (PHQ), visits to general practitioner, hospital readmissions and significant events involving major organ systems (thromboembolic; respiratory (including the Medical Research Council Respiratory questionnaire, use of long-term oxygen) and renal sequelae).

\section{Data management, data protection and patient confidentiality}

All investigators and trial site staff will comply with the requirements of General Data Protection Regulation with regard to the collection, storage, processing and disclosure of personal information and will uphold the Data Protection Act's core principles.

Where personal information is collected, it will be kept secure, and maintained. This will involve:
- The creation of coded, depersonalised data where the participant's identifying information is replaced by an unrelated sequence of characters.

- Secure maintenance of the data and the linking code in separate locations using encrypted digital files within password protected folders and storage media.

- Limiting access to the minimum number of individuals necessary for quality control, audit, and analysis.

- Confidentiality of data will be preserved when the data are transmitted to sponsors and coinvestigators as patients will be assigned a study ID number.

- Data collected from sites will be via the electronic database.

- Data custodian is Dr Jonathan Hewitt.

- Only researchers who are directly involved in the data analysis will have full access to the study data set (KM, BC, PB, SM, JH, PKM).

\section{Statistical analysis and sample size}

We will aim to recruit 500 patients; 50 patients across at least 10 sites. We estimated a minimum of $30 \%$ mortality in those that were frail, and $20 \%$ in those not frail (HR $0.60)$. In order to detect this difference with $80 \%$ power and with a $5 \%$ significance at least 500 patients are needed to detect this difference. However, due to the emergent nature of this condition, it is envisaged that an interim analysis will be conducted as sample size increases.

Statistical support will be provided by OPSOC. Data will be analysed for correlation between frailty and outcomes, including day 7 and day 90 mortality.

Our primary analyses will measure mortality as the time to mortality using a mixed effects Cox proportional hazards model, fitting site as a shared frailty (random intercept) to account for heterogeneity across different hospital sites.

Coprimary analyses will include: mortality at the short term (day 7), analysed with a mixed effects logistic regression at day 7 mortality.

The secondary outcome: Time-to-discharge (herein described as length of stay) analyses as a time-to-event analysis consistent with the primary analysis.

Secondary analyses will be adjusted for age group and gender, and other clinically important covariates. Other clinically important covariates will be included after discussion with the COPE investigators, once more information is known about the link between baseline covariates and their association with COVID-19 (eg, comorbidities). We will carry out a secondary analysis of the primary outcome by including additional clinical mediators which are determined clinically important and presented as a final multivariable model. All analyses will be presented as adjusted OR with associated 95\% CIs and $\mathrm{p}$ values. All other outcomes will be analysed as per the above analysis, but will be deemed secondary outcomes.

Longer term time points (eg, day 90) to be determined by the COPE investigators on receipt of further information from COVID-19 investigation. 
The time to mortality (or discharge) will be recorded from the date of admission to the date of outcome and will be considered community acquired. Where a patient has a positive COVID-19 diagnosis that is reported 15 days after admission (or later), they will be recorded as the date from diagnosis to outcome and considered as nosocomial infection.

Missing data will be explored for symptomatic rationale and may be imputed after discussion with the COPE investigators. Subgroups for age, sex and comorbidities may be explored to explain any mechanism of action found.

Anticipated recruitment data will be collected at participating sites for all patients meeting the inclusion criteria over a 3-month period. Validation will be performed by local teams on $25 \%$ of data fields for $10 \%$ of cases. The validated fields will include key demographic and outcome data.

\section{Study Management Group}

This will be led by PKM (Aberdeen) and will involve reviewing safety data and, since the study collates, observational data, and there are minimal safety issues expected.

\section{Patient and public involvement}

Due to the rapid onset of this pandemic and the urgency in analysis and dissemination of findings that will influence management of patients with COVID-19, it will not be possible to develop a patient and public involvement group of patients who have survived this illness. Many of them will remain clinically unwell, or in a period of selfisolation following COVID-19 infection. Towards the end of the study, if medically appropriate, the sponsor site will invite a group of patients back to the hospital to discuss their experiences. This will enable identification of key areas into which further investigation is required.

\section{Role of study sponsor and funder}

Aneurin Bevan NHS Trust, as the honorary NHS employer of the chief investigator (CI), has a critical role as part of the governance board of this project. Cardiff University (the CI's substantive employer) is the sponsor of the study. The sponsor is accountable for ensuring that the work is governed effectively and delivers the objectives that meet identified needs.

The sponsor will control the final decision regarding the study design, conduct, manuscript writing and dissemination of results.

The study at the time of writing has not received funding.

\section{Quality assurance and indemnity}

The quality of this study has been assessed by the following means:

- Establishment of the SMG.

- Peer review by professionals with relevant expertise (clinical trialists, nurses, front-line medical staff, statisticians, surgeons and geriatricians).

- Review by team at Cardiff University (sponsor institution).
- Safety reporting/adverse events will be reported by PIs. However, as this study involved routinely collected hospital data, we do not predict any.

This is an NHS-sponsored research study, therefore indemnity cover via Health Services Guidelines (96) 48 applies.

\section{Ethics and dissemination}

Ethical approval for the study was sought via proportional ethical review. However, data collected are routinely recorded clinical data. Therefore, the study was deemed not to require ethical approval and approval for the study was granted by the Health Research Authority (HRA) in the UK (https://www.hra.nhs.uk/) .

Subsequently, this study has been registered, reviewed and approved by the following organisations:

- The HRA for sites in England (20/HRA1898).

- The NHS Research Scotland Permissions Co-ordinating Centre (NRSPCC) for sites in Scotland, with reciprocal approval granted in Scotland by NRSPCC on 20 April 2020.

- The Health and Care Research Permissions Service for sites in Wales.

- Ethics Committee of Hospital Policlinico Modena, Italy (369/2020/OSS/AOUMO).

All participating units must obtain approval from their local Research and Development department consistent with the guidance from their relevant national organisation.

The project will therefore be registered locally with the NHS Trust or Health Board or Institutional Research and Development department prior to commencing patient identification and data collection at each site. It is the responsibility of the local COPE study team to ensure that local Research and Development approvals are in place prior to commencing data collection.

All data will be reported as a whole cohort. Unit-level data for comparison will be fed back to collaborators to support local service improvement. This project will be submitted for presentation at a national or international surgical and geriatric conference. Manuscript(s) will be prepared following the close of the project. Manuscripts will also be prepared following interim data analysis if numbers of patients recruited to the study at that time exceed the stated sample size.

\section{Author affiliations}

${ }^{1}$ Ageing and Complex Medicine, Salford Royal NHS Trust, Salford, UK

${ }^{2}$ Department of Anaesthetics, Royal Alexandra Hospital, Paisley, UK

${ }^{3}$ General Surgery, Royal Alexandra Hospital, Paisley, UK

${ }^{4}$ General Surgery, Salford Royal NHS Foundation Trust, Salford, UK

${ }^{5}$ University of Glasgow, Glasgow, UK

${ }^{6}$ Cardiovascular and Medical Sciences, University of Glasgow, Glasgow, UK

${ }^{7}$ General Surgery, University Hospital of Wales, Cardiff, UK

${ }^{8}$ Medicine for Older People, North Bristol NHS Trust, Westbury on Trym, UK

${ }^{9}$ University of Aberdeen, Aberdeen, UK

${ }^{10}$ Hospital Policlinico Modena, Modena, Italy

${ }^{11}$ General Surgery, North Bristol NHS Trust, Bristol, United Kingdom

${ }^{12}$ Biostatistics and Health Informatics, King's College London, London, UK

${ }^{13}$ King's College London, London, UK 
${ }^{14}$ Geriatric Medicine, Cardiff University, Cardiff, UK

Twitter Angeline Price @angeline_price, Susan Moug @susanmoug and Ben Carter @drbencarter

Collaborators COPE Study Collaborators: Charlotte Davey; Sheila Jones; Kiah Lunstone; Alice Cavenagh; Charlotte Silver; Thomas Telford; Rebecca Simmons; Sandeep Singh; Dolcie Paxton; Francis Rickard; Mark Holloway; James Hesford; Tarik Jichi; Norman Galbraith; Jenny Edwards; Emma Bhatti; Carly Bisset; Ross Alexander; Abigail Ingham; Roxanna Short; Aine McGovern; Jemima Collins; Eilidh Bruce; Alice Einarsson; Enrico Clini; Giovanni Guaraldi; Madeline Garcia; Shefali Sangani; Thomas Kneen; Thomas Lee; George Kyriakopoulos; Michael Thomas; Denise Tan.

Contributors All authors have read and agree to the finalised submitted version of the manuscript. Conceptualisation: JH, PKM. Methodology: JH, PKM, KM, BC. Software: BC. Validation: BC, JH, PKM. Formal analysis: BC. Investigation: AP, FBP, SD, AVM, SM, TQ, MS, PB, EM, PKM, AV, JH, KM, LP. Resources: JH, PKM. Data curation: BC. Writing — original draft preparation: AP, FBP, SD, AVM, SM, BC, LP. Writing—review and editing: all authors. Visualisation: all authors. Project administration: JH, PKM

Funding The authors have not declared a specific grant for this research from any funding agency in the public, commercial or not-for-profit sectors.

Competing interests None declared.

Patient consent for publication Not required.

Provenance and peer review Not commissioned; externally peer reviewed.

Open access This is an open access article distributed in accordance with the Creative Commons Attribution Non Commercial (CC BY-NC 4.0) license, which permits others to distribute, remix, adapt, build upon this work non-commercially, and license their derivative works on different terms, provided the original work is properly cited, appropriate credit is given, any changes made indicated, and the use is non-commercial. See: http://creativecommons.org/licenses/by-nc/4.0/.

\section{ORCID iDs}

Angeline Price http://orcid.org/0000-0002-4674-270X

Phyo Kyaw Myint http://orcid.org/0000-0003-3852-6158

Jonathan Hewitt http://orcid.org/0000-0002-7924-1792

\section{REFERENCES}

1 World Health Organization (WHO). WHO director-general's opening remarks at the media briefing on COVID-19. Available: https:// www.who.int/dg/speeches/detail/who-director-general-s-openingremarks-at-the-media-briefing-on-covid-19-11-march-2020 [Accessed 09 May 2020].

2 World Health Organization. Clinical management of severe acute respiratory infection (SARI) when COVID-19 disease is suspected. Available: https://www.who.int/publications-detail/clinicalmanagement-of-severe-acute-respiratory-infection-when-novelcoronavirus-(ncov)-infection-is-suspected [Accessed 09 May 2020].

3 O'Hanlon S, Inouye SK. Delirium: a missing piece in the COVID-19 pandemic puzzle. Age Ageing 2020;49:497-8.

4 Lithander FE, Neumann S, Tenison E, et al. COVID-19 in older people: a rapid clinical review. Age Ageing 2020;49:501-15.

5 Yang J, Zheng Y, Gou X, et al. Prevalence of comorbidities and its effects in patients infected with SARS-CoV-2: a systematic review and meta-analysis. Int J Infect Dis 2020;94:91-9.

6 Ticinesi A, Nouvenne A, Prati B, et al. Profiling the hospitaldependent patient in a large academic hospital: observational study. Eur J Intern Med 2019;64:41-7.

7 Fang L, Karakiulakis G, Roth M. Are patients with hypertension and diabetes mellitus at increased risk for COVID-19 infection? Lancet Respir Med 2020;8:e21.

8 Ingraham NE, Lofti-Emran S, Thielen B, et al. Immunomodulation in Covid-19. Lancet Respir Med 2020.

9 Royal College of General Practitioners. COVID-19 FAQs. Available: https://www.rcgp.org.uk/covid-19/covid-19-faqs.aspx [Accessed 09 May 2020].
10 British Society of Rheumatologists. Covid-19 guidance for rheumatologists, 2020. Available: https://www.rheumatology.org. uk/News-Policy/Details/Covid19-Coronavirus-update-members [Accessed 09 May 2020].

11 McDermid RC, Stelfox HT, Bagshaw SM. Frailty in the critically ill: a novel concept. Crit Care 2011;15:301.

12 Clegg A, Young J, lliffe S, et al. Frailty in elderly people. Lancet 2013;381:752-62.

13 Fried LP, Tangen CM, Walston J, et al. Frailty in older adults: evidence for a phenotype. J Gerontol A Biol Sci Med Sci 2001;56:M146-56.

14 Rockwood K, Song X, MacKnight C, et al. A global clinical measure of fitness and frailty in elderly people. CMAJ 2005;173:489-95.

15 Gilbert T, Neuburger J, Kraindler J, et al. Development and validation of a hospital frailty risk score focusing on older people in acute care settings using electronic hospital records: an observational study. Lancet 2018;391:1775-82.

16 Hewitt J, Moug SJ, Middleton M, et al. Prevalence of frailty and its association with mortality in general surgery. Am J Surg 2015;209:254-9.

17 Hewitt J, Carter B, McCarthy K, et al. Frailty predicts mortality in all emergency surgical admissions regardless of age. An observational study. Age Ageing 2019;48:388-94.

18 Vilches-Moraga A, Rowley M, Fox J, et al. Emergency laparotomy in the older patient: factors predictive of 12-month mortality-SalfordPOPS-GS. An observational study. Aging Clin Exp Res 2020. doi:10.1007/s40520-020-01578-0. [Epub ahead of print: 24 May 2020].

19 Bebb O, Smith FG, Clegg A, et al. Frailty and acute coronary syndrome: a structured literature review. Eur Heart J Acute Cardiovasc Care 2018;7:166-75.

20 Walker DM, Gale CP, Lip G, Martin-Sanchez FJ, et al. Editor's Choice - Frailty and the management of patients with acute cardiovascular disease: A position paper from the Acute Cardiovascular Care Association. Eur Heart J Acute Cardiovasc Care 2018;7:176-93.

21 Royal College of Surgeons of England. The high-risk general surgica patient: raising the standard, 2018. Available: https://www.surgeons. org/media/25768745/rcs-report-the-highrisk-general-surgicalpatient-raising-thestandard-december-2018.pdf [Accessed 10 May 2020].

22 Cesari M, Costa N, Hoogendijk EO, et al. How the frailty index may support the allocation of health care resources: an example from the INCUR study. J Am Med Dir Assoc 2016;17:448-50.

23 Muscedere J, Waters B, Varambally A, et al. The impact of frailty on intensive care unit outcomes: a systematic review and meta-analysis. Intensive Care Med 2017;43:1105-22.

24 NICE. COVID-19 rapid guideline: critical care in adults NICE guideline, 2020. Available: www.nice.org.uk/guidance/ng159

25 Guan W-J, Ni Z-Y, Hu Y, et al. Clinical characteristics of coronavirus disease 2019 in China. N Engl J Med 2020;382:1708-20.

26 Richardson S, Hirsch JS, Narasimhan M, et al. Presenting characteristics, comorbidities, and outcomes among 5700 patients hospitalized with COVID-19 in the new York City area. JAMA 2020;323:2052

27 Zhou F, Yu T, Du R, et al. Clinical course and risk factors for mortality of adult inpatients with COVID-19 in Wuhan, China: a retrospective cohort study. Lancet 2020;395:1054-62.

28 ICNARC. The ICNARC report on covid-19 in critical care, 2020. Available: https://www.icnarc.org/Our-Audit/Audits/Cmp/Reports

29 Parmar KL, Law J, Carter B, et al. Frailty in older patients undergoing emergency laparotomy. Ann Surg 2019

30 Carter B, Law J, Hewitt J, et al. Association between preadmission frailty and care level at discharge in older adults undergoing emergency laparotomy. Br J Surg 2020;107:218-26.

31 Hewitt J, Owen S, Carter BR, et al. The prevalence of delirium in an older acute surgical population and its effect on outcome. Geriatrics 2019;4:57.

32 Myint PK, Owen S, McCarthy K, et al. Is anemia associated with cognitive impairment and delirium among older acute surgical patients? Geriatr Gerontol Int 2018;18:1025-30.

33 Dowswell G, Bartlett DC, Futaba K, et al. How to set up and manage a trainee-led research collaborative. BMC Med Educ 2014;14:94.

34 Jelley B, Long S, Butler J, et al. Cohort profile: the Welsh geriatric registrar-led research network $(\mathrm{WeGeN})$ : rationale, design and description. BMJ Open 2017;7:e013031. 